\title{
24
}

\section{Catalytic Control of Architecture and Properties of Butadiene Block Copolymers}

\author{
PH. TEYSSIE, G. BROZE, R. FAYT, J. HEUSCHEN, R. JEROME, \\ and D. PETIT
}

University of Liège, Laboratory of Macromolecular Chemistry and Organic

Catalysis, Sart-Tilman, 4000 Liège, Belgium

It is shown how a precise control of the initiation and selectivity

of butadiene block polymerization reactions allows the molecular engineering of a variety of new heterophase materials. The typical examples presented illustrate 3 main potentialities of these copolymers. First, the stable emulsification of blends of 2 immiscible homopolymers (PE/PS; PVC/PBD) by the corresponding diblock copolymer. Second, the possibility to obtain high performance thermoplastic elastomers from triblock copolymers containing endblocks of high cohesive energy density (i.e. nylon-6, polypivalolactone). Third, the opportunity to tailor "multiblock" systems in which the "hard" segment is an ionic group (halatotelechelic polymers), imparting to these systems interesting rheological properties.

Catalytic polymerizations of 1,3-butadiene into essentially 1,4 elastomeric polymers are by now pretty well-mastered processes. It is the purpose of this paper to demonstrate the still very vivid interest in that time-honored backbone, within the frame of a much broader trend developing now in the field of polymeric materials.

It is obvious indeed that industry is not willing anymore to produce many of these necessary materials from new monomers; the key approach here is thus diversification, but starting from basic feedstocks and materials already available at low prices in large amounts. On the 
other hand, in a time of explosive development of "genetic engineering" techniques in biology, it is only too fair to acknowledge a similar type of achievement in polymeric materials science, i.e. the "molecular engineering of their bulk properties". That recent capability to achieve, through precise (although sometimes small) modifications of molecular structures, a "finetuning" of the final bulk properties and macroscopic behaviour of these polymeric materials, has arisen from our rapidly increasing knowledge and mastering of initiation and propagation mechanisms, particularly in terms of selectivity. That allows us in turn to control accurately the molecular architecture of the polymers used, that means also the morphology and further the physico-chemical as well as physico-mechanical behaviour of the corresponding final materials.

All of the examples presented here, leading to different types of properties and applications, are based on copolymers of varying architecture, but all involving essentially 1,4 polybutadiene blocks. Clearly, their properties will thus reflect three important potentialities of heterophase block copolymer-based materials : 1) their "organization" into mesomorphic phases (1), giving rise to original, often anisotropic, properties; 2) their ability to stabilize liquid emulsions (2) and more importantly fine dispersions of corresponding homopolymers ( 3 ), so bridging their "compatibility gap" and allowing the development of a new "plasturgy"; 3) their use as high-performance engineering products, and particularly as materials similar to Kraton thermoplastic elastomers ( $\underline{4})$.

Experimental

All of the important experimental details, as well as the general procedures taken from literature, were reported in the references cited in each section. As a general rule, most of the reagents were purified carefully (particularly for moisture), and all of the reactions carried out either using vacuum techniques or under pure argon atmosphere. Polymer properties were investigated by standard methods, unless otherwise mentioned. Polymer blending was usually performed for 5 minutes at $190^{\circ} \mathrm{C}$ on a C.A.M.I.L. two-roll laboratory mill.

$\underline{\text { Results }}$

A_preliminary_problelem. The unsaturated structure of the 1,4 polybutadiene $(P B D)$ backbone is unfortunately 
rather sensitive to a number of easily occurring secondary reactions, and particularly to different types of cross-linking and oxidation processes (thermal, photochemical, and catalytic). This drawback has been elegantly circumvented by a facile catalytic hydrogenation of the chains, using efficient soluble Ziegler-type complexes (5) prepared from cobalt (or nickel) salts and mixed aluminum alkyls in different ratios. This process has a twofold advantage :

1) it is possible to control its selectivity (varying the reaction conditions and the aluminum-transition metal molar ratio), so that polybutadiene only can be hydrogenated in the presence of other unsaturated chains like polyisoprene or polystyrene, which in turn can be hydrogenated later, if necessary by changing these reaction parameters;

2) the hydrogenation is practically quantitative and yields a product not very different from the corresponding polyethylenes. For instance, starting from an anionically initiated polybutadiene (in apolar medium) containing accordingly ca. 10-15 1,2 units, one obtains a product rather similar to low density linear polyethylene LLDPE (in fact a copolymer of ethylene and 1-butene), displaying a moderate semi-crystallinity and a melting point $\mathrm{Ca} 90$ to $100^{\circ} \mathrm{C}$. On the other hand, the use of a linear pure 1,4 PBD (99\%) obtained by Zieglertype catalysis (nickel or cobalt complexes) yields a high-density polyethylene-like product, with a higher degree of crystallinity and a melting-point close to $130^{\circ} \mathrm{C}$.

Obviously, the resistance of these products towards light, oxygen and other chemicals will be much better, and close to that of the corresponding polyolefins. Moreover, the hydrogenation can be stopped at different conversions opening a much broader range of applications conditions. Industrial developments already include successful materials like Kraton $G$ thermoplastic elastomers.

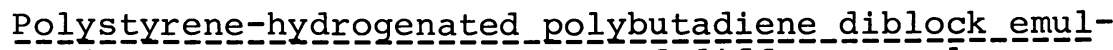

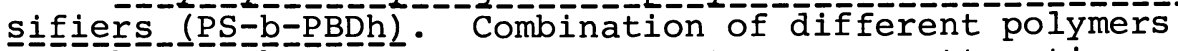
into he terophase systems represents a very attractive route towards new and tailor-made materials (3) displaying most of the properties of the starting products. The incompatibility between partners (a rather general rule) is however responsible for the poor properties of many blends, which display large domain size with poor interfacial adhesion. Accordingly, diblock copolymers (6) containing sequences miscible with the homopolymers to be blended, have been used to alleviate that situation as a 
result of their interfacial activity; in that respect, they have proven to be superior (7) to corresponding triblock and graft copolymers respectively (unless the triblock can undergo a tridimensional domain organization providing an additional bonus).

Two different types of (PS-b-PBDh) diblock can be presently synthesized. The first one by classical anionic initiation (s-butyl-lithium) and "living" propagation of the (PS-b-PBD) copolymer (8), followed by the hydrogenation procedure described here; as discussed above, the resulting product will be close to a (PS-bLLDPE) copolymer. The second one came from the discovery (9) of a "living" polymerization of butadiene into a pure (99 \%) 1, 4 polymer by a bis $n^{3}$-allylnickel-trifluoroacetate) coordination catalyst, followed by styrene polymerization; unfortunately, the length of the polystyrene block is limited (to a M.W. of ca. 20,000) by transfer reactions.

In a general study of the usefulness of the first type copolymers, we have developed ways to drastically improve with them the properties of both low (LD) and high (HD) density polyethylene (PE)/PS blends $(7,10)$. The addition (by hot-milling) of moderate amounts of the suitable PS-b-PDBh copolymer greatly reduces the dispersed particles size at every composition (ca. 2to 5,000 A); moreover, it stabilizes efficiently that situation, i.e. even throughout standard processing. A significant enhancement of both the stress at break $\sigma_{B}$ and the elongation at break $\varepsilon_{B}$, resulting in a stri$\mathrm{king}$ increase of the total energy at break ( $E_{\mathrm{B}}$ values), is noted (Fig.l).

The level of performance obtained depends asymptotically on the amount of copolymer added and, while a significant improvement is already noted for $0.5 \%$ by weight of the additive, most of the performances increase is reached within 2-3 \%. As expected, the length of the two blocks has also a great influence on the final properties : although smaller ones already have a great impact on the interfacial situation (smaller domains, higher $\sigma_{B}$ ), the use of higher M.W. diblocks (i.e. in which each block is similar in size to the corresponding homopolymers to be blended) yields PS-rich blends displaying the mechanical characteristics of an excellent toughened plastic, with a high $\varepsilon_{B}$ value (up to $40 \%)$ a typically ductile behaviour, and a striking resistance towards cryofracture. These features are probably characteristic of the importance of entanglements between chains of homo- and copolymer near the interface.

Interesting and significant differences are also promo- 

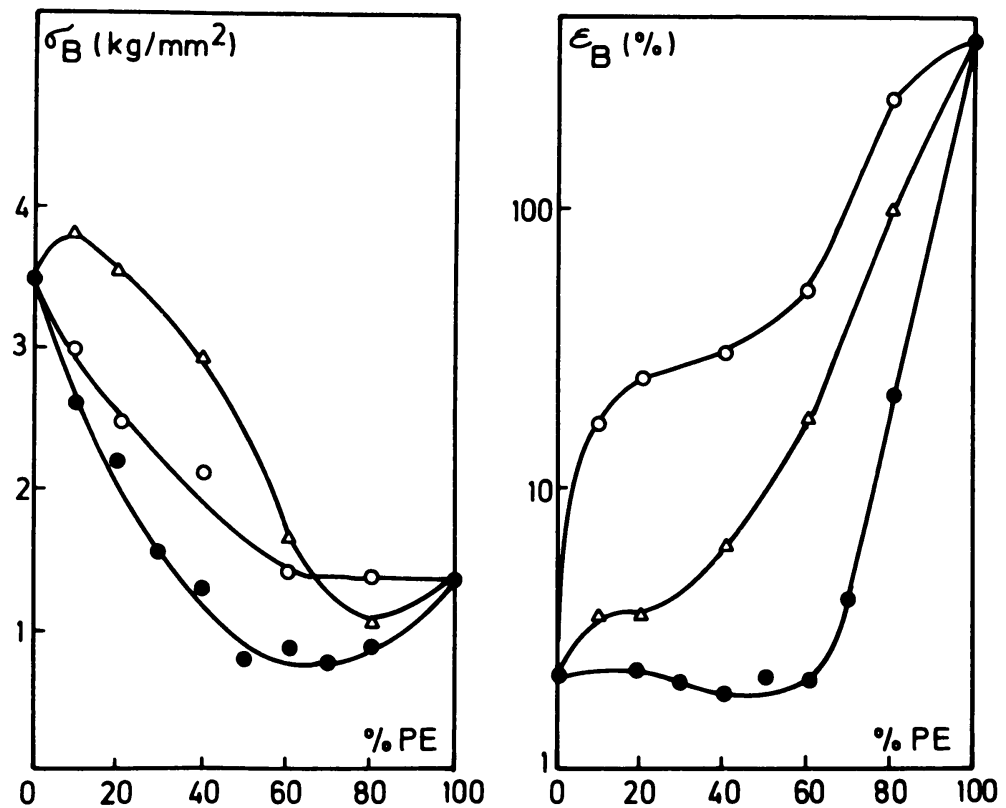

Figure 1. Ultimate tensile strength $\left(\sigma_{B}\right)$ and elongation at break $\left(\epsilon_{B}\right)$ of LDPE $\left(\mathrm{M}_{\mathrm{n}}=40,000\right) / P S\left(\mathrm{M}_{\mathrm{n}}=10^{5}\right)$ blends. Key: 0 , without copolymer; $\triangle$, with $9 \%$ of a poly(styrene-b-hydrogenated butadiene), $\mathrm{M}_{\mathrm{n}}$ total $=58,000 ; 0$, with $9 \%$ of a poly(styrene-b-hydrogenated butadiene), $\mathbf{M}_{\mathrm{n}}$ total $=155,000$. (Reproduced with permission from Ref. 7. Copyright 1981, John Wiley \& Sons, Inc.) 
ted by rather subtle modifications in the diblock structure. Compared to "pure" diblocks prepared by consecutive anionic polymerization, "tapered" blocks obtained by anionic polymerization of the comonomers mixture (11) are still more efficient emulsifiers for PS/LDPE blends; owing to their lower melt viscosity and particular miscibility characteristics, they not only act as solubilizing agents of the homopolymers but provide at the interface a "graded" modulus responsible for an improved mechanical response of the overall material (12).

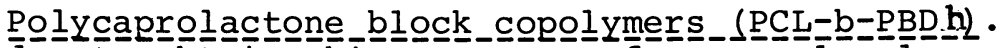
In order to obtain this new type of macromolecule, an OH-terminated homopolymer, i.e. PBD or PS obtained by anionic initiation combined with oxirane-water termination, was end-capped (an alcohol displacement reaction) with an alkoxide catalyst able to further ensure a perfectly "living" polymerization of CL, thereby yielding the desired block copolymers (13). In this case however the PBD hydrogenation (if needed) can be conducted prior to block copolymerization to avoid any interference from, or secondary reaction with, the polyester block. The final products display a number of attractive features (14): they undergo lamellar mesomorphic organization (periodicity ca. $80 \AA$ ) even under the form of hexagonal single crystals (PS-copolymers), and exhibit a ductile behaviour as well as a high resistance to cryofracture. Moreover, they are macroscopically biodegradable, at least when PCL represents the continous phase.

The blending approach described

above has been extended to these products, taking advantage of the remarkable miscibility of PCL with other polymers, i.e. PVC, SAN, polycarbonate .... In particular, excellent blends of rigid PVC with PS and PBD have been prepared through hot-milling. Again, they display a very fine morphology (domain size ca $5,000 \AA$ ) which is remarkably stable in time, and some improved physicomechanical properties as long as the corresponding molecular parameters have been properly optimized $(15,16)$. It should be stressed here that the need for such an optimization process cannot be over-estimated as rather minute modifications can lead to improvements of one order of magnitude.

Other interesting indications have been obtained on these PVC blends : in the presence of an excess rubbery phase, the presence of the copolymer (i.e. PCL-bPBD) promotes indeed good impact resistance, indicating a strong anchorage of that rubbery phase in the resin matrix. 
In other words, this example emphasizes clearly the very broad applicability of the "emulsifiers" concept depicced above, to practical problems involving largescale polymers.

The synthesis of high-performance thermoplastic el as tomer The interest of TP has been largely demonstrat by the numerous and active investigations led in the field, and by the commercial success of the Kraton-type products, poly (styrene-co-diene-co-sytrene). It is however a well-known problem that these materials are usually confined to applications under rather mild conditions, due to the relatively low $\mathrm{T}_{\mathrm{g}}$ of the glassy phase and/or the mediocre thermal stability of the rubbery phase. In principle at least, answers to that challenge can be offered by the same type of synthesis strategy.

As described previously (17), an

$\alpha, \omega$-dihydroxylpolybutadiene can be end-capped with isocyanate functions (through reaction with an excess diisocyanate), which are further converted into N-acyllactam groupings (by reaction with caprolactam CLM) active for the polymerization of that CLM monomer into Nylon-6 blocks at both ends of the polybutadiene. Under close control of the reaction conditions, neither the urethane nor the urea linkages formed are broken during the subsequent block copolymerization, and a high yield in block copolymer is obtained. That triblock copolymer displays indeed a set of high performance properties : even at $30 \%$ of PBD phase, the material has a nylon-6 continuous phase, with a crystalline melting point ca. $225^{\circ} \mathrm{C}$ and a high tensile strength (quite comparable to that of pure nylon-6). On the other hand, the rubbery phase is finely dispersed in very small domains (ca. $250 \AA)$ all over the highly crystalline nylon phase,imparting to the material higher flexibility and hydrophobicity. Although the above product is not a TPE in the strict sense of the term (see ASTM D 1566), it can be prepared with a higher rubber content to meet that type of behaviour.

It has to be stressed however that blocks of so different solubility parameters impose a heterophase situation with strong intermolecular interactions (on the polar side), even in the molten state well above the crystalline melting point : corresponding stained micrographs are very informative in that respect (see ref. (17), fig. 4d). Such a situation raises obviously severe rheological processing problems, which might be difficult to solve. Anyhow, it implies also to work at a temperature which is exceedingly detrimental for the 
unsaturated 1,4-polybutadiene block; again, preliminary quantitative hydrogenation of the starting polybutadiene is the answer to that problem, even though the difunctional macromolecules form a highly swollen gel in the presence of an excess catalyst.

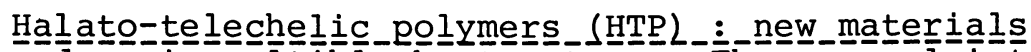
with_a a dynamíc.mu rest of multibiock copolymers particularly in the field of TPE. Based on the efficient cross-linking action of the harder blocks, interesting developments have taken place, leading to some successful industrial materials such as HYTREL polyetherester thermoplastic elastomers. The very rapid developments of increasingly sophisticated catalysts has also promoted new unexpected achievements; a typical example (18) is the "coding" 1,4 polymerization of butadiene by "tailored" catalysts, namely bis ( $n^{3}$-allylnickel -X) complexes. Under precisely controlled kinetic conditions, a multistereoblock (poly.cis 1,4-b-poly.trans $1,4)_{n}$-butadiene can be obtained, that represents the first example of a thermoplastic elastomer (semicrystalline melting point ca. $135^{\circ} \mathrm{C}$ ) obtained in one step from one single monomer.

A completely different approach has been recently developed in that prospect, based on the assumption that "properties similar to those of multiblock copolymers could be reached, in a more versatile manner, by replacing their hard segments by single groupings, provided the molecular characteristics of these groupings promote very strong mutual interactions, at least in the media envisoned for their applications".

That concept had led to the synthesis of so-called "halato-telechelic polymers" (which means a "salt" or "neutralized" telechelic polymer, acidic or basic). Although that is a very general denomination covering all the chains formed by any type of ion-pair coupling in any way, a particularly handy and representative class of such structures can be obtained from the complete neutralization of $\alpha, \omega$-dicarboxylato-polymers (PX), by a di (or multi-) valent metal derivative, (19), according to the general equation :

$\mathrm{n} \quad \mathrm{HOOC}-\mathrm{PX}-\mathrm{COOH}+\frac{2 \mathrm{n}}{\mathrm{v}} \mathrm{MA}_{\mathrm{v}} \leqq \mathrm{FOOC}^{\mathrm{P} X}-\mathrm{COO}-\mathrm{M}_{\frac{2}{\mathrm{v}}} \mathrm{f} \overline{\mathrm{n}}+2 \mathrm{nHA}$

Such a reaction has been performed successfully, starting from anionically prepared telechelic polymers PX, and neutralizing them quantitatively with very reactive 
metal derivatives such as metal alkyls or alkoxides (20); the latter technique proved to be the most versatile one, provided a complete elimination of the alcohol evolved in order to displace the reaction but also to avoid solvation by that alcohol : that meets the essential requirement of any stepwise polymerization, i.e. the necessity to ensure a very high (99\%+) conversion to reach the high degree of polymerization necessary for the promotion of the most interesting physical properties, that in turn would be overshadowed if ion solvation by the alcohol takes place (see below). In that way, a broad family of HTP has been synthesized, wherein the nature and size of both the polymer and the ion involved can be systematically modified, as well as that of the solvent and ligands. They represent accordingly a versatile class of materials with a broad potential range of "molecularly engineered" characteristics. The most_typical_property of hydrocararbon-soluble HTP (i.e., where PX is a polydiene or PS, or polyisobutene) with a high enough $M W(>1,000)$ to avoid excessive charge density), is the strong dependence of its dilute-solution viscosity upon concentration (20) . Although very similar to that of PX at very low concentrations, it increases abruptly and asymptotically between ca. 1 and $2 \%$, resulting in a gelation_phenome= non. This gelation can usually be reduced by incréasing the temperature, or upon addition of strong ligands (Fig. 2). The critical gel concentration, $\mathrm{C}_{\mathrm{g}}$, depends essentially on the nature of $\mathrm{PX}$, its end-groups, the solvent, the cation size, and on $\mathrm{PX}$ molecular weight following the relationship $\mathrm{C}_{\mathrm{g}}=\mathrm{k} \cdot \overline{\mathrm{M}}_{\mathrm{n}}^{-8.5}$ (where $\mathrm{k}^{-1 / 3}$ is directly proportional to $\left[\frac{(\mathrm{r} 2)}{\mathrm{M}}\right]^{0.5}, \mathrm{i} . \mathrm{n}$, depends

\section{on the mean end-to-end distance of the free chain)}

These are ofcourse clear-cut manifestations of the electrostatic interactions between ion-pairs in a non-polar solvent, leading to aggregates of variable sizes depending on the conditions; for the same reasons, the same type of intermolecular association will obviously occur in the neat material. It is also noteworthy that such a "multiblock" structure is a dynamic one, i.e., carboxylic ligands exchanging around the metal ion resulting in a constant scrambling not only of the ionic aggregates but also of the chain blocks themselves. That situation is in fact responsible for the dynamic mechanical properties of these materials.

With_concentrated solutions (over 50 - $\%$ ) at_equilibrium as wèlì as with the neat products, ${ }^{\prime} i \bar{t}$ is possib̄lé to observe typical SAXS patterns, often exhibiting two diffraction orders with Bragg's spacing in 1:2 ratio sug- 


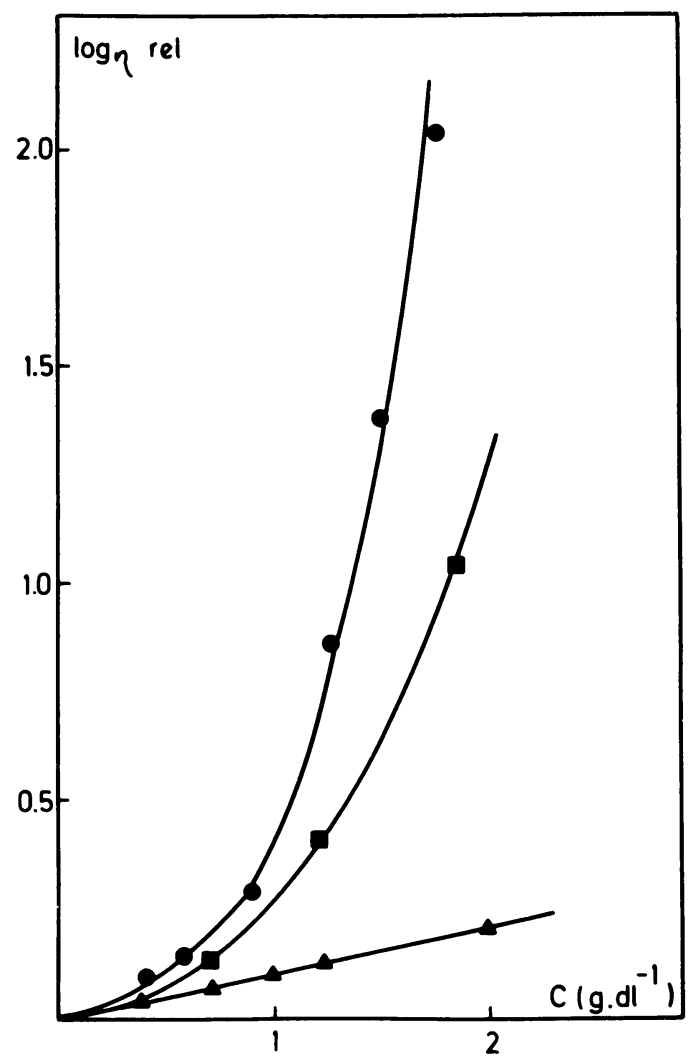

Figure 2. Relative viscosity-concentration plots in toluene. Key: $\boldsymbol{\Delta}$, nonneutralized $\alpha, \omega$-dicarboxylic PBD $\left(\mathrm{M}_{\mathrm{n}}=4,600\right) ; 0, \mathrm{Mg}$ salt of $\alpha, \omega$-dicarboxylic $P B D, 25^{\circ} \mathrm{C}$;

$\square, M g$ salt of $\alpha, \omega$-dicarboxylic $P B D, 80^{\circ} \mathrm{C}$. 
gesting the presence of a lamellar organization (22). The calculated lamellar thickness seems independent of the nature of the ion as well as of temperature (below a critical dissociation value), but again strongly reflects the mean dimensions of the free chains. The rheological behaviour of these products has also been ${ }^{-}$investigate $\bar{d}(\underline{2} \overline{3})$. The steady-flow viscosity depends markedly on the shear-rate, and for values higher

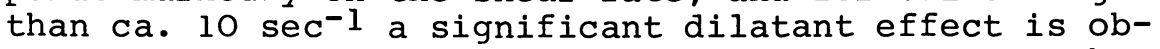
served, since preformed ionic cross-links prevent the chains to relax as the deformation time-scale decreases. In_terms_of dynamic mechanical_behaviour, storage (G') and $\bar{l}$ loss ${ }^{-}\left(\bar{G}^{\prime}\right)$ moduli ${ }^{-}$have been détermined (23) over a range of frequencies for different polymers. At higher frequency (>0.5 sec. $\left.{ }^{-1}\right), G^{\prime}$ is higher than G", which in turn presents a maximum characteristic of the ionic component relaxation mechanism. These elastic gels have relaxation spectra which indicate again the ions aggregation : a broad distribution of relaxation times appears, the maximum of which depends on the stability of the ionic network. Since these materials display a nice thermorheological simplicity (at least when $\mathrm{P}_{\mathrm{X}}$ has a $\overline{\mathrm{M}}_{n}$ lower than 20,000, i.e., no entanglements interference), master curves have been established. The corresponding shift factors correlate nicely in an Arrhenius-type relationship, allowing the determination of the activation energy of the secondary relaxation mechanism. For different ions, these energies are directly dependent on the ion electrostatic field.

In other_words, these properties can be interpreted in terms of a system in which the deformation processes are governed by ionic multiplets'thermal dissociation; however, while electrostatic attractive forces are determinant in the aggregation process, the free conformation of the macromolecule will control the overall morphology of the gels as well as the mean number of ions in the multiplets.

Several interesting applications can be envisioned on the basis of that behaviour, but again saturatedelastomers will be more suitable for most of them, in terms of ageing. Here again, hydrogenated telechelic polybutadiene is a possible answer to that problem, although telechelic polyisobutene might alternatively be used in some cases.

General_Conclusion. At this point, it is probably useful and certain $\bar{y}$ encouraging to stress again that all of these examples confirm the versatility of the molecular engineering techniques presently available, as well as their potentialities in broadening and diver- 
sifying the applications of such a time-honored commodity polymer as polybutadiene. That is certainly a worthwhile goal in today's technology and economy.

\section{Literature Cited}

1. Sadron, C.; Gallot, B. Makromo1. Chem. 1973, 164, 301; Gallot, B. "Liquid Crystalline Order in Polymers," Blumstein, A, Ed.; Academic: New York, $1978 ; 191$.

2. Marti, S.; Nervo, J.; Riess, G. Progr. Colloid. Polym. Sci. $1975,58,114$.

3. Paul, D.R. "Polymer B̈lends," Paul,D.R. and Newman, S., Eds.; Academic: New York, 1978; 168.

4. Holden, G.; Bishop, E.T.; Legge, N.R. J. Polymer Sci. $1969, \mathrm{C} 36,37$.

5. Falk, J.C. J.Polym. Sci. 1971, A-1,9, 2617; Gillies, G.A. (to Shell Oil Co.), U.S. Patent $3,792,127$ (1974).

6. Fayt, R.; Jérôme, R. Actualite Chimique $1980,21$.

7. Fayt, R.; Jérôme, R.; Teyssié, Ph. J. Polym. Sci. Polym. Lett. Ed. 1981, 19, 79 .

8. Szwarc, M. "Carbanions, Living Polymers and Electron-Transfer Processes," and references therein, Wiley-Interscience: New York, 1968.

9. Hadjiandreau, P. Ph.D., Thesis, University of Liege, Liege, 1980.

10. Fayt, R.; Jérôme, R.; Teyssié, Ph. J. Polym. Sci. Polym. Phys. Ed. 1981, 19, 1269.

11. Kuntz, I. J. Polym. Sci. 1961, 54, 569.

12. Fayt, R.; Jérôme, R.; Teyssié, $\overline{\mathrm{Ph}}$. J. Polym. Sci. Polym. Phys. Ed. (in press).

13. Heuschen, J.; Jerome, R.; Teyssie, Ph. Macromolecules 1981, 14, 242.

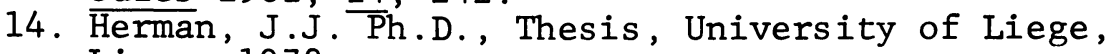
Liege, 1978.

15. Teyssié, Ph.; Bioul, J.P.; Hamitou, A.; Heuschen, J.; Hocks, L.; Jéróme, R.; Ouhadi, T. ACS Symposium Series No.59, T. Saegusa and E. Goethals Eds., 1977.

16. Heuschen, J.; Jérồme, R.; Teyssié, Ph. French Patent 2,383,208 (1977); U.S.Patent 4,281,087(1981).

17. Petit. D.; Jérôme, R.; Teyssié, Ph. J. Polym. Sci. Polym. Chem. Ed. 1979, 17, 2903.

18. Teyssie, Ph.; Devaux, A.; Hadjiandreou, P.; Julémont, M.; Thomassin, J.M.; Walckiers, E.; Ouhadi, T. "Preparation and Properties of Stereoregular Polymers," Lenz, R.W. and Ciardelli, F., Eds.; M. Reidel: Hingham, Mass., 1980; 144.

19. Moudden, A.; Levelut, A.M.; Pineri, M. J. Polym. Sci. Polym. Lett. Ed. 1977, 15, 1707. 
20. Broze, G.; Jérôme, R.; Teyssié, Ph. Macromolecules (in press) and references therein.

21. Broze, G.; Jérôme, R.; Teyssié, Ph. Macromolecules (in press).

22. Gallot, B. ; Broze, G.; Jérôme, R.; Teyssié, Ph. J. Polym. Sci. Polym. Lett. Ed. 1981, 19, 415.

23. Broze, G.; Jérồme, R.; Teyssie, Ph. Polym. Bull. 1981, 4, 241; Part VII, J. Polym. Sci. Polym. Phys. Ed. (to be published).

ReCEIVEd December 23, 1982 